\title{
Wavelet approach to operator-valued Hardy spaces
}

\author{
Guixiang Hong and Zhi Yin
}

\begin{abstract}
This paper is devoted to the study of operator-valued Hardy spaces via the wavelet method. This approach is parallel to that in the noncommutative martingale case. We show that our Hardy spaces defined by wavelets coincide with those introduced by Tao Mei via the usual Lusin and Littlewood-Paley square functions. As a consequence, we give an explicit complete unconditional basis of the Hardy space $H_{1}(\mathbb{R})$ when $H_{1}(\mathbb{R})$ is equipped with an appropriate operator space structure.
\end{abstract}

\section{Introduction}

In this paper, we exploit Meyer's wavelet methods to study operator-valued Hardy spaces. We are motivated by two rapidly developing fields. The first one is the theory of noncommutative martingale inequalities. This theory was initiated already in the 1970's. Its modern period of development began with Pisier and Xu's seminal paper [20], in which the authors established the noncommutative Burkholder-Gundy inequalities and the Fefferman duality theorem between $H_{1}$ and BMO. Since then many classical results have been successfully transferred to the noncommutative world (see [11], [14], [15], [1]). In particular, motivated by [9], Mei [15] developed the theory of Hardy spaces on $\mathbb{R}^{n}$ for operator-valued functions.

Our second motivation is the theory of wavelets founded by Meyer. It is now well known that this theory is important for many domains, in particular in harmonic analysis. For instance, it provides powerful tools for the theory of CalderónZygmund singular integral operators. More recently, Meyer's wavelet methods were extended to study more sophisticated subjects in harmonic analysis. For example, the authors of [5] exploited the properties of Meyer's wavelets to give a characterization of product BMO by commutators; [17] deals with the estimates of bi-parameter paraproducts.

Mathematics Subject Classification (2010): Primary 46L52; Secondary 42B30, 42B35, 42C40, 46B70, 46M35.

Keywords: Noncommutative $L_{p}$ spaces, Hardy spaces, BMO spaces, wavelets, duality, interpolation. 
It is in this spirit that we wish to understand how useful wavelet methods are for noncommutative analysis. The most natural and possible way would be first to do this in the semicommutative case. This is exactly the purpose of the present paper which could be viewed as the first attempt at developing wavelet techniques for noncommutative analysis.

A wavelet basis of $L_{2}(\mathbb{R})$ is a complete orthonormal system $\left(w_{I}\right)_{I \in \mathcal{D}}$, where $\mathcal{D}$ denotes the collection of all dyadic intervals in $\mathbb{R}, w$ is a Schwartz function satisfying the properties needed for Meyer's construction in [16], and

$$
w_{I}(x):=\frac{1}{|I|^{\frac{1}{2}}} w\left(\frac{x-c_{I}}{|I|}\right),
$$

where $c_{I}$ is the center of $I$. The central facts that we will need about wavelet bases are the orthogonality between different $w_{I}$ 's, that $\|w\|_{L_{2}(\mathbb{R})}=1$, and the regularity of $w$,

$$
\max \left(|w(x)|,\left|w^{\prime}(x)\right|\right) \precsim(1+|x|)^{-m}, \quad \forall m \geq 2 .
$$

The analogy between wavelets and dyadic martingales is well known. The key observation is the following parallelism:

$$
\sum_{|I|=2^{-n+1}}\left\langle f, w_{I}\right\rangle w_{I} \sim d f_{n},
$$

where $d f_{n}$ denotes the $n$-th dyadic martingale difference of $f$. This parallelism indicates that martingale methods may be used to deal with wavelets. With this in mind, we develop the theory of operator-valued Hardy spaces, which are defined through wavelets, in the way that is well known in the noncommutative martingale case. Then we show that our Hardy and BMO spaces coincide with Mei's.

This paper is organized as follows. In Section 1, we will give some preliminaries on noncommutative analysis, and the definitions in our setting of $\mathcal{H}_{p}(\mathbb{R}, \mathcal{M})$ with $1 \leq p<\infty$ and $L_{q} \mathcal{M O}(\mathbb{R}, \mathcal{M})$ with $2<q \leq \infty$. In Section 2 , we are concerned with three duality results. The most important one is the noncommutative analogue of the famous Fefferman duality theorem between $\mathcal{H}_{1}^{c}(\mathbb{R}, \mathcal{M})$ and $\mathcal{B M O}^{c}(\mathbb{R}, \mathcal{M})$. The second is the duality between $\mathcal{H}_{p}^{c}(\mathbb{R}, \mathcal{M})$ and $L_{p^{\prime}}^{c} \mathcal{M O}(\mathbb{R}, \mathcal{M})$ with $1<p<2$, where we need a noncommutative version of Doob's inequality. This is why we consider the case $1<p<2$ independently. The last one is the duality between $\mathcal{H}_{p}^{c}(\mathbb{R}, \mathcal{M})$ and $\mathcal{H}_{p^{\prime}}^{c}(\mathbb{R}, \mathcal{M})$ when $1<p<\infty$. As a corollary of the last two results, we identify $\mathcal{H}_{q}^{c}(\mathbb{R}, \mathcal{M})$ and $L_{q}^{c} \mathcal{M O}(\mathbb{R}, \mathcal{M})$ with $2<q<\infty$. Section 3 deals with interpolation for our Hardy spaces. In the last section, we show that our Hardy spaces coincide with those of [15]. Consequently, we can give an explicit completely unconditional basis for the space $H_{1}(\mathbb{R})$, when $H_{1}(\mathbb{R})$ is equipped with an appropriate operator space structure.

We end this introduction by introducing the convention that, throughout the paper, $c$ will denote an absolute positive constant, which may vary from line to line, and $c_{p}$ will denote a positive constant depending only on $p$. 


\section{Preliminaries}

\subsection{Operator-valued noncommutative $L_{p}$-spaces}

Let $\mathcal{M}$ be a von Neumann algebra equipped with a normal semifinite faithful trace $\tau$ and let $S_{\mathcal{M}}^{+}$be the set of all positive elements $x$ in $\mathcal{M}$ with $\tau(s(x))<\infty$, where $s(x)$ is the smallest projection $e$ such that $e x e=x$. Let $S_{\mathcal{M}}$ be the linear span of $S_{\mathcal{M}}^{+}$. Then any $x \in S_{\mathcal{M}}$ has finite trace, and $S_{\mathcal{M}}$ is a $w^{*}$-dense $*$-subalgebra of $\mathcal{M}$.

Let $1 \leq p<\infty$. For any $x \in S_{\mathcal{M}}$, the operator $|x|^{p}$ belongs to $S_{\mathcal{M}}^{+}(|x|=$ $\left.\left(x^{*} x\right)^{\frac{1}{2}}\right)$. We define

$$
\|x\|_{p}=\left(\tau\left(|x|^{p}\right)\right)^{1 / p}, \quad \forall x \in S_{\mathcal{M}}
$$

One can check that $\|\cdot\|_{p}$ is well defined and is a norm on $S_{\mathcal{M}}$. The completion of $\left(S_{\mathcal{M}},\|\cdot\|_{p}\right)$ is denoted by $L_{p}(\mathcal{M})$ which is the usual noncommutative $L_{p^{-}}$space associated with $(\mathcal{M}, \tau)$. For convenience, we usually set $L_{\infty}(\mathcal{M})=\mathcal{M}$ equipped with the operator norm $\|\cdot\|_{\mathcal{M}}$. The elements of $L_{p}(\mathcal{M}, \tau)$ can be described as closed densely defined operators on $H$ ( $H$ being the Hilbert space on which $\mathcal{M}$ acts). We refer the reader to [21] for more information on noncommutative $L_{p}$-spaces.

In this paper, we are concerned with three operator-valued noncommutative $L_{p}$-spaces. The first one is the noncommutative space $L_{p}\left(\mathcal{M} ; \ell_{2}^{c}\right)\left(\operatorname{resp.} L_{p}\left(\mathcal{M} ; \ell_{2}^{r}\right)\right)$, which is studied at length in [9]. For this space, we need the following properties. In the sequel, $p^{\prime}$ will always denote the conjugate index of $p$.

Lemma 2.1. Let $1 \leq p<\infty$. Then

$$
\left(L_{p}\left(\mathcal{M} ; \ell_{2}^{c}\right)\right)^{*}=L_{p^{\prime}}\left(\mathcal{M} ; \ell_{2}^{c}\right)
$$

Thus, for $f=\left(f_{k}\right)_{k} \in L_{p}\left(\mathcal{M} ; \ell_{2}^{c}\right)$ and $g=\left(g_{k}\right)_{k} \in L_{p^{\prime}}\left(\mathcal{M} ; \ell_{2}^{c}\right)$, we have

$$
|\tau(\langle f, g\rangle)| \leq\|f\|_{L_{p}\left(\mathcal{M} ; \ell_{2}^{c}\right)}\|g\|_{L_{p^{\prime}}\left(\mathcal{M} ; \ell_{2}^{c}\right)},
$$

where

$$
\langle f, g\rangle=\sum_{k} f_{k} g_{k}^{*}
$$

Lemma 2.2. Let $1 \leq p_{0}<p<p_{1} \leq \infty, 0<\theta<1, \frac{1}{p}=\frac{1-\theta}{p_{0}}+\frac{\theta}{p_{1}}$. Then

$$
\left[L_{p_{0}}\left(\mathcal{M} ; \ell_{2}^{c}\right), L_{p_{1}}\left(\mathcal{M} ; \ell_{2}^{c}\right)\right]_{\theta}=L_{p}\left(\mathcal{M} ; \ell_{2}^{c}\right)
$$

A similar equality holds for row spaces.

The second space is the $\ell_{\infty}$-valued noncommutative space $L_{p}\left(\mathcal{M} ; \ell_{\infty}\right)$, which is studied by Pisier [19] for an injective $\mathcal{M}$, and by Junge [8] for a general $\mathcal{M}$ (see also [11] and [13] for more properties). For this space, we need the following property: 
Lemma 2.3. Let $1 \leq p<\infty$. Then

$$
\left(L_{p}\left(\mathcal{M} ; \ell_{1}\right)\right)^{*}=L_{p^{\prime}}\left(\mathcal{M} ; \ell_{\infty}\right) .
$$

Thus, for $x=\left(x_{n}\right)_{n} \in L_{p}\left(\mathcal{M} ; \ell_{1}\right)$ and $y=\left(y_{n}\right)_{n} \in L_{p^{\prime}}\left(\mathcal{M} ; \ell_{\infty}\right)$, we have

$$
\left|\sum_{n \geq 1} \tau\left(x_{n} y_{n}\right)\right| \leq\|x\|_{L_{p}\left(\mathcal{M} ; \ell_{1}\right)}\|y\|_{L_{p^{\prime}}\left(\mathcal{M} ; \ell_{\infty}\right)},
$$

where $L_{p}\left(\mathcal{M} ; \ell_{1}\right)$ is the space of all sequences $x=\left(x_{n}\right)_{n}$ such that

$$
\left\|\left(x_{k}\right)_{k}\right\|_{L_{p}\left(\mathcal{N} ; \ell_{1}\right)}=\sup _{x_{n}=\sum_{k} a_{n, k}^{*} b_{n, k}}\left\|\sum_{n, k}\left|a_{n, k}\right|^{2}\right\|_{p}^{1 / 2}\left\|\sum_{n, k}\left|b_{n, k}\right|^{2}\right\|_{p}^{1 / 2} .
$$

The third space is $L_{p}\left(\mathcal{M} ; \ell_{\infty}^{c}\right)$ for $2 \leq p \leq \infty$, which was introduced in [4] and is related with the second space by

$$
\left\|\left(x_{n}\right)_{n}\right\|_{L_{p}\left(\mathcal{M} ; \ell_{\infty}^{c}\right)}=\left\|\left(\left|x_{n}\right|^{2}\right)_{n}\right\|_{L_{\frac{p}{2}}\left(\mathcal{M} ; \ell_{\infty}\right)} .
$$

These are normed spaces for the following characterization of the norm:

$$
\left\|\left(x_{n}\right)_{n}\right\|_{L_{p}\left(\mathcal{M} ; \ell_{\infty}^{c}\right)}=\inf _{x_{n}=y_{n} a}\left\|\left(y_{n}\right)\right\|_{\ell_{\infty}\left(L_{\infty}(\mathcal{M})\right)}\|a\|_{L_{p}(\mathcal{M})}
$$

We need the following interpolation results for these spaces (see [18]):

Lemma 2.4. Let $2 \leq p_{0}<p<p_{1} \leq \infty, 0<\theta<1$, and $\frac{1}{p}=\frac{1-\theta}{p_{0}}+\frac{\theta}{p_{1}}$. Then

$$
\left[L_{p_{0}}\left(\mathcal{M} ; \ell_{\infty}^{c}\right), L_{p_{1}}\left(\mathcal{M} ; \ell_{\infty}^{c}\right)\right]_{\theta}=L_{p}\left(\mathcal{M} ; \ell_{\infty}^{c}\right)
$$

\subsection{Operator-valued Hardy spaces}

In this paper, for simplicity, we denote $L_{\infty}(\mathbb{R}) \bar{\otimes} \mathcal{M}$ by $\mathcal{N}$. As indicated in the introduction, one can observe that we have the operator-valued Calderón identity

$$
f(x)=\sum_{I \in \mathcal{D}}\left\langle f, w_{I}\right\rangle w_{I}(x)=\sum_{I \in \mathcal{D}} \int_{\mathbb{R}} f(y) \overline{w_{I}(y)} d y w_{I}(x),
$$

which holds when $f \in L_{2}(\mathcal{N})$. As in the classical case, for $f \in S_{\mathcal{N}}$, we define the two Littlewood-Paley square functions as

$$
\begin{aligned}
& S_{c}(f)(x)=\left(\sum_{I \in \mathcal{D}} \frac{\left|\left\langle f, w_{I}\right\rangle\right|^{2}}{|I|} \mathbb{1}_{I}(x)\right)^{\frac{1}{2}}, \\
& S_{r}(f)(x)=\left(\sum_{I \in \mathcal{D}} \frac{\left|\left\langle f^{*}, w_{I}\right\rangle\right|^{2}}{|I|} \mathbb{1}_{I}(x)\right)^{\frac{1}{2}} .
\end{aligned}
$$

For $1 \leq p<\infty$, define

$$
\|f\|_{\mathcal{H}_{p}^{c}}=\left\|S_{c}(f)\right\|_{L_{p}(\mathcal{N})} \quad \text { and } \quad\|f\|_{\mathcal{H}_{p}^{r}}=\left\|S_{r}(f)\right\|_{L_{p}(\mathcal{N})} .
$$


These are norms, which can be seen easily from the space $L_{p}\left(\mathcal{N} ; \ell_{2}^{c}(\mathcal{D})\right)$. So we define the space $\mathcal{H}_{p}^{c}(\mathbb{R}, \mathcal{M})$ (resp., $\mathcal{H}_{p}^{r}(\mathbb{R}, \mathcal{M})$ ) as the completion of $\left(S_{\mathcal{N}},\|\cdot\|_{\mathcal{H}_{p}^{c}}\right)$ (resp., $\left(S_{\mathcal{N}},\|\cdot\|_{\mathcal{H}_{p}^{r}}\right)$.

Now, we define the operator-valued Hardy spaces as follows: for $1 \leq p<2$,

$$
\mathcal{H}_{p}(\mathbb{R}, \mathcal{M})=\mathcal{H}_{p}^{c}(\mathbb{R}, \mathcal{M})+\mathcal{H}_{p}^{r}(\mathbb{R}, \mathcal{M})
$$

with the norm

$$
\|f\|_{\mathcal{H}_{p}}=\inf \left\{\|g\|_{\mathcal{H}_{p}^{c}}+\|h\|_{\mathcal{H}_{p}^{r}}: f=g+h, g \in \mathcal{H}_{p}^{c}, h \in \mathcal{H}_{p}^{r}\right\},
$$

and for $2 \leq p<\infty$,

$$
\mathcal{H}_{p}(\mathbb{R}, \mathcal{M})=\mathcal{H}_{p}^{c}(\mathbb{R}, \mathcal{M}) \cap \mathcal{H}_{p}^{r}(\mathbb{R}, \mathcal{M})
$$

with the norm

$$
\|f\|_{\mathcal{H}_{p}}=\max \left\{\|f\|_{\mathcal{H}_{p}^{c}},\|f\|_{\mathcal{H}_{p}^{r}}\right\} .
$$

We can identify $\mathcal{H}_{p}^{c}(\mathbb{R}, \mathcal{M})$ as a subspace of $L_{p}\left(\mathcal{N} ; \ell_{2}^{c}(\mathcal{D})\right)$, which is related with the two maps below:

Definition 2.5. (i) The map $\Phi$ is defined from $\mathcal{H}_{p}^{c}(\mathbb{R}, \mathcal{M})$ to $L_{p}\left(\mathcal{N} ; \ell_{2}^{c}(\mathcal{D})\right)$ by

$$
\Phi(f)=\left(\frac{\left\langle f, w_{I}\right\rangle}{|I|^{\frac{1}{2}}} \mathbb{1}_{I}\right)_{I \in \mathcal{D}} .
$$

(ii) The projection map $\Psi$ is defined from $L_{2}\left(\mathcal{N} ; \ell_{2}^{c}(\mathcal{D})\right)$ to $\mathcal{H}_{2}^{c}(\mathbb{R}, \mathcal{M})$ by

$$
\Psi\left(\left(g_{I}\right)\right)=\sum_{I \in \mathcal{D}} \int \frac{g_{I}}{|I|^{\frac{1}{2}}} \mathbb{1}_{I} d y \cdot w_{I} .
$$

\subsection{Operator-valued $\mathcal{B M O}$ spaces}

For $\varphi \in L_{\infty}\left(\mathcal{M} ; L_{2}^{c}\left(\mathbb{R}, \frac{d x}{1+x^{2}}\right)\right)$, set

$$
\begin{aligned}
\|\varphi\|_{\mathcal{B M O}^{c}} & =\sup _{J \in \mathcal{D}}\left\|\left(\frac{1}{|J|} \sum_{I \subset J}\left|\left\langle\varphi, w_{I}\right\rangle\right|^{2}\right)^{\frac{1}{2}}\right\|_{\mathcal{M}} \text { and } \\
\|\varphi\|_{\mathcal{B M O}^{r}} & =\left\|\varphi^{*}\right\|_{\mathcal{B M} \mathcal{O}^{c}(\mathbb{R}, \mathcal{M})} .
\end{aligned}
$$

These are again norms modulo constant functions. Define

$$
\begin{aligned}
& \mathcal{B M O}^{c}(\mathbb{R}, \mathcal{M})=\left\{\varphi \in L_{\infty}\left(\mathcal{M} ; L_{2}^{c}\left(\mathbb{R}, \frac{d x}{1+x^{2}}\right)\right):\|\varphi\|_{\mathcal{B M O}^{c}}<\infty\right\}, \quad \text { and } \\
& \mathcal{B M O}^{r}(\mathbb{R}, \mathcal{M})=\left\{\varphi \in L_{\infty}\left(\mathcal{M} ; L_{2}^{r}\left(\mathbb{R}, \frac{d x}{1+x^{2}}\right)\right):\|\varphi\|_{\mathcal{B M O}^{r}}<\infty\right\} .
\end{aligned}
$$

Now we define

$$
\mathcal{B M O}(\mathbb{R}, \mathcal{M})=\mathcal{B M O}^{c}(\mathbb{R}, \mathcal{M}) \cap \mathcal{B M O}^{r}(\mathbb{R}, \mathcal{M})
$$


As in the martingale case [11], we can also define $L_{p}^{c} \mathcal{M O}(\mathbb{R}, \mathcal{M})$ for all $2<p \leq \infty$. For $\varphi \in L_{p}\left(\mathcal{M} ; L_{2}^{c}\left(\mathbb{R}, \frac{d x}{1+x^{2}}\right)\right)$, set

$$
\|\varphi\|_{L_{p}^{c} \mathcal{M O}}=\left\|\left(\frac{1}{\left|I_{k}^{x}\right|} \sum_{I \subset I_{k}^{x}}\left|\left\langle\varphi, w_{I}\right\rangle\right|^{2}\right)_{k}\right\|_{L_{\frac{p}{2}}\left(\mathcal{N} ; \ell_{\infty}\right)}^{\frac{1}{2}}
$$

and

$$
\|\varphi\|_{L_{p}^{r} \mathcal{M O}}=\left\|\varphi^{*}\right\|_{L_{p}^{c} \mathcal{M O O}}
$$

where $I_{k}^{x}$ denotes the unique dyadic interval with length $2^{-k+1}$ that contains $x$. We will use the convention adopted in [13] for the norm in $L_{\frac{p}{2}}\left(\mathcal{N} ; \ell_{\infty}\right)$. Thus

$$
\left\|\left(\frac{1}{\left|I_{k}^{x}\right|} \sum_{I \subset I_{k}^{x}}\left|\left\langle\varphi, w_{I}\right\rangle\right|^{2}\right)_{k}\right\|_{L_{\frac{p}{2}}\left(\mathcal{N} ; \ell_{\infty}\right)}^{\frac{1}{2}}=\left\|\sup _{k}^{+} \frac{1}{\left|I_{k}^{x}\right|} \sum_{I \subset I_{k}^{x}}\left|\left\langle\varphi, w_{I}\right\rangle\right|^{2}\right\|_{L_{\frac{p}{2}(\mathcal{N})}}^{\frac{1}{2}}
$$

These are norms, which follow from the Banach spaces $L_{p}\left(\mathcal{N} \bar{\otimes} B\left(\ell_{2}(\mathcal{D})\right) ; \ell_{\infty}^{c}\right)$. Again, we can define

$$
\begin{aligned}
& L_{p}^{c} \mathcal{M O}(\mathbb{R}, \mathcal{M})=\left\{\varphi \in L_{p}\left(\mathcal{M} ; L_{2}^{c}\left(\mathbb{R}, \frac{d x}{1+x^{2}}\right)\right):\|\varphi\|_{L_{p}^{c} \mathcal{M O}}<\infty\right\}, \quad \text { and } \\
& L_{p}^{r} \mathcal{M O}(\mathbb{R}, \mathcal{M})=\left\{\varphi \in L_{p}\left(\mathcal{M} ; L_{2}^{r}\left(\mathbb{R}, \frac{d x}{1+x^{2}}\right)\right):\|\varphi\|_{L_{r}^{c} \mathcal{M O}}<\infty\right\} .
\end{aligned}
$$

Define

$$
L_{p} \mathcal{M O}(\mathbb{R}, \mathcal{M})=L_{p}^{c} \mathcal{M O}(\mathbb{R}, \mathcal{M}) \cap L_{p}^{r} \mathcal{M O}(\mathbb{R}, \mathcal{M}) .
$$

Note that $L_{\infty}^{c} \mathcal{M O}(\mathbb{R}, \mathcal{M})=\mathcal{B M O}^{c}(\mathbb{R}, \mathcal{M})$. It is easy to check that all the spaces we have defined here are Banach spaces with respect to the relevant norms.

\section{Duality}

To prove the first two duality results in this section, we need the following noncommutative version of Doob's inequality from [8].

Let $\left(\mathcal{E}_{n}\right)_{n}$ be the conditional expectation with respect to a filtration $\left(\mathcal{N}_{n}\right)_{n}$ of $\mathcal{N}$.

Lemma 3.1. Let $1<p \leq \infty$ and $f \in L_{p}(\mathcal{N})$. Then

$$
\left\|\sup _{n}+\mathcal{E}_{n}(f)\right\|_{L_{p}(\mathcal{N})} \leq c_{p}\|f\|_{L_{p}(\mathcal{N})}
$$

Theorem 3.2. We have

$$
\left(\mathcal{H}_{1}^{c}(\mathbb{R}, \mathcal{M})\right)^{*}=\mathcal{B M O}^{c}(\mathbb{R}, \mathcal{M})
$$

with equivalent norms. That is, every $\varphi \in \mathcal{B M O}^{c}(\mathbb{R}, \mathcal{M})$ induces a continuous linear functional $l_{\varphi}$ on $\mathcal{H}_{1}^{c}(\mathbb{R}, \mathcal{M})$ by

$$
l_{\varphi}(f)=\tau \int \varphi^{*} f, \quad \forall f \in S_{\mathcal{N}}
$$


Conversely, for every $l \in\left(\mathcal{H}_{1}^{c}(\mathbb{R}, \mathcal{M})\right)^{*}$, there exists a $\varphi \in \mathcal{B M O}^{c}(\mathbb{R}, \mathcal{M})$ such that $l=l_{\varphi}$. Moreover,

$$
c^{-1}\|\varphi\|_{\mathcal{B M O}^{c}} \leq\left\|l_{\varphi}\right\|_{\left(\mathcal{H}_{1}^{c}\right)^{*}} \leq c\|\varphi\|_{\mathcal{B M O}^{c}}
$$

where $c>0$ is a universal constant.

Similarly, duality holds between $\mathcal{H}_{1}^{r}$ and $\mathcal{B M O}^{r}$, and between $\mathcal{H}_{1}$ and $\mathcal{B M O}$, with equivalent norms.

In order to adapt the arguments in the martingale case, we need to define the truncated square functions for $n \in \mathbb{Z}$,

$$
S_{c, n}(f)(x)=\left(\sum_{k=-\infty}^{n} \sum_{|I|=2^{-k+1}} \frac{\left|\left\langle f, w_{I}\right\rangle\right|^{2}}{|I|} \mathbb{1}_{I}(x)\right)^{\frac{1}{2}} .
$$

Proof. Since $S_{\mathcal{N}}$ is dense in $\mathcal{H}_{1}^{c}(\mathbb{R}, \mathcal{M})$, by an approximation argument we only need to prove the inequality

$$
\left|l_{\varphi}(f)\right| \leq c\|\varphi\|_{\mathcal{B M O}^{c}}\|f\|_{\mathcal{H}_{1}^{c}}
$$

for $f \in S_{\mathcal{N}}$. By approximation we may assume that $S_{c, n}(f)(x)$ is invertible in $\mathcal{M}$ for all $x \in \mathbb{R}$ and $n \in \mathbb{Z}$. Then we have

$$
\begin{aligned}
\left|l_{\varphi}(f)\right| & =\left|\tau \int \varphi^{*} f d x\right|=\left|\sum_{n} \tau \int \sum_{|I|=2^{-n+1}}\left\langle\varphi, w_{I}\right\rangle^{*} w_{I} \sum_{\left|I^{\prime}\right|=2^{-n+1}}\left\langle f, w_{I^{\prime}}\right\rangle w_{I^{\prime}} d x\right| \\
& =\left|\sum_{n} \tau \int \sum_{|I|=2^{-n+1}} \frac{\left\langle\varphi, w_{I}\right\rangle^{*}}{|I|^{\frac{1}{2}}} \mathbb{1}_{I} \sum_{\left|I^{\prime}\right|=2^{-n+1}} \frac{\left\langle f, w_{I^{\prime}}\right\rangle}{|I|^{\frac{1}{2}}} \mathbb{1}_{I^{\prime}} d x\right| \\
& \leq \sum_{n}\left(\tau \int\left|\sum_{|I|=2^{-n+1}} \frac{\left\langle f, w_{I}\right\rangle}{|I|^{\frac{1}{2}}} \mathbb{1}_{I}\right|^{2} S_{c, n}^{-1}(f)\right)^{\frac{1}{2}} \\
& \leq\left(\sum_{n} \tau \int \sum_{|I|=2^{-n+1}} \frac{\left|\left\langle f, w_{I}\right\rangle\right|^{2}}{|I|} \mathbb{1}_{I} S_{c, n}^{-1}(f)\right)^{\frac{1}{2}} \\
& \left.\cdot\left(\sum_{n} \tau \sum_{|I|=2^{-n+1}} \sum_{|I|=2^{-n+1}} \frac{\left|\left\langle\varphi, w_{I}\right\rangle\right|^{2}}{|I|} \mathbb{1}_{I} S_{c, n}(f)\right)^{\frac{1}{2}} S_{c, n}(f)\right)^{\frac{1}{2}} \\
& =A \cdot B .
\end{aligned}
$$

In the above estimates, the second equality uses the orthogonality of the $w_{I}$ 's on different levels, and the third one the orthogonality of the $w_{I}$ 's on the same level and the disjointness of different dyadic I's on the same level; the first inequality uses the Hölder inequality of Lemma 2.1, and the second one uses the CauchySchwarz inequality and the disjointness of different I's on the same level. 
Now, let us estimate $A$ :

$$
\begin{aligned}
A^{2} & =\sum_{n} \tau \int\left(S_{c, n}^{2}(f)-S_{c, n-1}^{2}(f)\right) S_{c, n}^{-1}(f) \\
& =\sum_{n} \tau \int\left(S_{c, n}(f)-S_{c, n-1}(f)\right)\left(1+S_{c, n-1}(f) S_{c, n}^{-1}(f)\right) \\
& \leq \sum_{n} \tau \int\left(S_{c, n}(f)-S_{c, n-1}(f)\right)\left\|1+S_{c, n-1}(f) S_{c, n}^{-1}(f)\right\|_{\infty} \\
& \leq 2 \sum_{n} \tau \int\left(S_{c, n}(f)-S_{c, n-1}(f)\right)=2\|f\|_{\mathcal{H}_{1}^{c}} .
\end{aligned}
$$

For the first inequality, we have used the Hölder inequality and the positivity of $S_{c, n}(f)-S_{c, n-1}(f)$.

The second term is estimated as follows:

$$
\begin{aligned}
B^{2} & =\sum_{k} \tau \int\left(S_{c, k}(f)-S_{c, k-1}(f)\right) \sum_{n \geq k} \sum_{|I|=2^{-n+1}} \frac{\left|\left\langle\varphi, w_{I}\right\rangle\right|^{2}}{|I|} \mathbb{1}_{I} \\
& =\sum_{k} \tau \sum_{j}\left(S_{c, k}(f)-S_{c, k-1}(f)\right) \int_{I_{k}^{j}} \sum_{n \geq k} \sum_{|I|=2^{-n+1}} \frac{\left|\left\langle\varphi, w_{I}\right\rangle\right|^{2}}{|I|} \mathbb{1}_{I} \\
& =\sum_{k} \tau \sum_{j} \int_{I_{k}^{j}}\left(S_{c, k}(f)-S_{c, k-1}(f)\right) \frac{1}{\left|I_{k}^{j}\right|} \sum_{I \subset I_{k}^{j}}\left|\left\langle\varphi, w_{I}\right\rangle\right|^{2} \\
& \leq \sum_{k} \sum_{j} \tau \int_{I_{k}^{j}}\left(S_{c, k}(f)-S_{c, k-1}(f)\right)\left\|\frac{1}{\left|I_{k}^{j}\right|} \sum_{I \subset I_{k}^{j}}\left|\left\langle\varphi, w_{I}\right\rangle\right|^{2}\right\|_{\infty} \\
& \leq\|\varphi\|_{\mathcal{B} \mathcal{M O O}^{c}}^{2} \sum_{k} \sum_{j} \tau \int_{I_{k}^{j}}\left(S_{c, k}(f)-S_{c, k-1}(f)\right) \\
& =\|\varphi\|_{\mathcal{B} \mathcal{M O}^{c}}^{2}\|f\|_{\mathcal{H}_{1}^{c}} .
\end{aligned}
$$

We use, in the first equality, the Fubini theorem; and in the second equality, the fact that $S_{c, k-1}(f)$ and $S_{c, k}(f)$ are constant on the dyadic interval $I_{k}^{j}=\left[j 2^{-k+1}\right.$, $\left.(j+1) 2^{-k+1}\right)$. For the first inequality the Hölder inequality and the positivity of $S_{c, n}(f)-S_{c, n-1}(f)$ are used.

Now, let us begin to deal with the other direction, i.e., supposing that $l$ is a bounded linear functional on $\mathcal{H}_{1}^{c}(\mathbb{R}, \mathcal{M})$, we want to find an operator-valued function $\varphi$ in $\mathcal{B M O}^{c}(\mathbb{R}, \mathcal{M})$ such that $l=l_{\varphi}$ and $l_{\varphi}(f)=\tau \int \varphi^{*} f$ for $f \in S_{\mathcal{N}}$. By the embedding operator $\Phi$ in (2.10) and by the Hahn-Banach theorem, $l$ extends to a bounded continuous functional on $L_{1}\left(\mathcal{N} ; \ell_{2}^{c}(\mathcal{D})\right)$ of the same norm. Then, by Lemma 2.1, there exists $g=\left(g_{I}\right)_{I \in \mathcal{D}}$ such that $\|g\|_{L_{\infty}\left(\mathcal{N} ; \ell_{2}^{c}(\mathcal{D})\right)}=\|l\|$, and

$$
l(f)=\tau \int \sum_{I \in \mathcal{D}} g_{I}^{*} \frac{\left\langle f, w_{I}\right\rangle}{|I|^{\frac{1}{2}}} \mathbb{1}_{I}, \quad \forall f \in S_{\mathcal{N}} .
$$


Now let $\varphi=\Psi(g)$, where $\Psi$ is defined as (2.11). The orthogonality of the $w_{I}$ 's yields

$$
\begin{aligned}
\left\|\sum_{I \subset J}\left|\left\langle\varphi, w_{I}\right\rangle\right|^{2}\right\|_{\mathcal{M}} & =\left\|\sum_{I \subset J}\left|\int \frac{g_{I}}{|I|^{\frac{1}{2}}} \mathbb{1}_{I}\right|^{2}\right\|_{\mathcal{M}} \leq\left\|\sum_{I \subset J} \int_{J}\left|g_{I}\right|^{2}\right\|_{\mathcal{M}} \\
& \leq|J|\left\|\sum_{I \subset J}\left|g_{I}\right|^{2}\right\|_{L_{\infty}(\mathcal{N})} \leq|J|\left\|\left(g_{I}\right)_{I}\right\|_{L_{\infty}\left(\mathcal{N} ; \ell_{2}^{c}(\mathcal{D})\right)},
\end{aligned}
$$

where in the first inequality the Kadison-Schwarz inequality has been used. Also thanks to the orthogonality of the $w_{I}$ 's, we get

$$
l(f)=\tau \int \sum_{I \in \mathcal{D}} g_{I}^{*} \frac{\left\langle f, w_{I}\right\rangle}{|I|^{\frac{1}{2}}} \mathbb{1}_{I}=\tau \int \varphi^{*} f
$$

for all $f \in S_{\mathcal{N}}$. Therefore, we have completed the proof for the column spaces. Passing to adjoints, we have the conclusion concerning $\mathcal{H}_{1}^{r}$ and $\mathcal{B} \mathcal{M O}{ }^{r}$. Finally, by the classical fact that the dual of a sum space is the intersection space, we obtain the duality between $\mathcal{H}_{1}$ and $\mathcal{B M O}$.

Theorem 3.3. Let $1<p<2$. We have

$$
\left(\mathcal{H}_{p}^{c}(\mathbb{R}, \mathcal{M})\right)^{*}=L_{p^{\prime}}^{c} \mathcal{M O}(\mathbb{R}, \mathcal{M})
$$

with equivalent norms. That is, every $\varphi \in L_{p^{\prime}}^{c} \mathcal{M O}(\mathbb{R}, \mathcal{M})$ induces a continuous linear functional $l_{\varphi}$ on $\mathcal{H}_{p}^{c}(\mathbb{R}, \mathcal{M})$ by

$$
l_{\varphi}(f)=\tau \int \varphi^{*} f, \quad \forall f \in S_{\mathcal{N}}
$$

Conversely, for every $l \in\left(\mathcal{H}_{p}^{c}(\mathbb{R}, \mathcal{M})\right)^{*}$, there exists an operator-valued function $\varphi \in L_{p^{\prime}}^{c} \mathcal{M O}(\mathbb{R}, \mathcal{M})$ such that $l=l_{\varphi}$ and

$$
c_{p}^{-1}\|\varphi\|_{L_{p^{\prime}}^{c} \mathcal{M O}} \leq\left\|l_{\varphi}\right\|_{\left(\mathcal{H}_{p}^{c}\right)^{*}} \leq \sqrt{2}\|\varphi\|_{L_{p^{\prime}}^{c} \mathcal{M O}} .
$$

Similarly, duality holds between $\mathcal{H}_{p}^{r}$ and $L_{p^{\prime}}^{r}$, and between $\mathcal{H}_{p}$ and $L_{p^{\prime}} \mathcal{M O}$, with equivalent norms.

We need the following lemma from [11]. We recall it for the reader's convenience, but without proof.

Lemma 3.4. Let $s$ and $t$ be two real numbers such that $s<t$ and $0 \leq s \leq 1 \leq t \leq 2$. Let $x$ and $y$ be two positive operators such that $x \leq y$ and $x^{t-s}, y^{t-s} \in L_{1}(\mathcal{N})$. Then

$$
\tau \int y^{-s / 2}\left(y^{t}-x^{t}\right) y^{-s / 2} \leq 2 \tau \int y^{-(s+1-t) / 2}(y-x) y^{-(s+1-t) / 2}
$$


Proof of Theorem 3.3. We only need to prove the first assertion about $\mathcal{H}_{p}^{c}$. Since $S_{\mathcal{N}}$ is dense in $\mathcal{H}_{p}^{c}(\mathbb{R}, \mathcal{M})$, by an approximation argument, we only need to prove the inequality

$$
\left|l_{\varphi}(f)\right| \leq c\|\varphi\|_{L_{p^{\prime}}^{c} \mathcal{M O}}\|f\|_{\mathcal{H}_{p}^{c}}
$$

for $f \in S_{\mathcal{N}}$. By approximation we may assume that $S_{c, n}(f)(x)$ is invertible in $\mathcal{M}$ for all $x \in \mathbb{R}$ and $n \in \mathbb{Z}$. By the same principle as in the noncommutative martingale case as in [11], we have

$$
\begin{aligned}
\left|l_{\varphi}(f)\right|= & \left|\tau \int \varphi^{*} f d x\right|=\left|\sum_{n} \tau \int \sum_{|I|=2^{-n+1}}\left\langle\varphi, w_{I}\right\rangle^{*} w_{I} \sum_{\left|I^{\prime}\right|=2^{-n+1}}\left\langle f, w_{I^{\prime}}\right\rangle w_{I^{\prime}} d x\right| \\
= & \left|\sum_{n} \tau \int \sum_{|I|=2^{-n+1}} \frac{\left\langle\varphi, w_{I}\right\rangle^{*}}{|I|^{\frac{1}{2}}} \mathbb{1}_{I} \sum_{\left|I^{\prime}\right|=2^{-n+1}} \frac{\left\langle f, w_{I^{\prime}}\right\rangle}{|I|^{\frac{1}{2}}} \mathbb{1}_{I^{\prime}} d x\right| \\
\leq & \sum_{n}\left(\tau \int\left|\sum_{|I|=2^{-n+1}} \frac{\left\langle f, w_{I}\right\rangle}{|I|^{\frac{1}{2}}} \mathbb{1}_{I}\right|^{2} S_{c, n}^{p-2}(f)\right)^{\frac{1}{2}} \\
\leq & \left.\left.\cdot \sum_{n} \tau \int \sum_{|I|=2^{-n+1}} \frac{\mid\left\langle\varphi, w_{I}\right\rangle}{|I|^{\frac{1}{2}}} \mathbb{1}_{I}\right|^{2} S_{c, n}^{2-p}(f)\right)^{\frac{1}{2}} \\
& \cdot\left(\sum_{n} \tau \sum_{\left.|I|=2_{I}^{-n+1}\right\rangle\left.\right|^{2}} \mathbb{1}_{I} \frac{\left|\left\langle\varphi, w_{I}\right\rangle\right|^{2}}{|I| n} \mathbb{1}_{I} S_{c, n}^{2-p}(f)\right)^{\frac{1}{2}} \\
= & A \cdot B .
\end{aligned}
$$

Now we need Lemma 3.4 to estimate the first term. Take $s=2-p$ and $t=2$. The lemma yields

$$
\begin{aligned}
A^{2} & =\sum_{n} \tau \int\left(S_{c, n}^{2}(f)-S_{c, n-1}^{2}(f)\right) S_{c, n}^{p-2}(f) \\
& =\sum_{n} \tau \int S_{c, n}^{-(2-p) / 2}(f)\left(S_{c, n}^{2}(f)-S_{c, n-1}^{2}(f)\right) S_{c, n}^{-(2-p) / 2}(f) \\
& \leq 2 \sum_{n} \tau \int S_{c, n}^{-(1-p) / 2}(f)\left(S_{c, n}(f)-S_{c, n-1}(f)\right) S_{c, n}^{-(1-p) / 2}(f) \\
& =2 \sum_{n} \tau \int S_{c, n}(f)-S_{c, n-1}(f) S_{c, n}^{p-1}(f) \\
& \leq 2 \sum_{n}^{n} \tau \int S_{c, n}^{p}(f)-S_{c, n-1}^{p}(f) \\
& =2\|f\|_{\mathcal{H}_{p}^{c}}^{p} .
\end{aligned}
$$

The last inequality has used two elementary inequalities: $0 \leq S_{c, n-1}(f) \leq S_{c, n}(f)$ implies $S_{c, n-1}^{p-1}(f) \leq S_{c, n}^{p-1}(f)$ for $0<p-1<1$; and

$$
\tau\left(S_{c, n-1}^{p}(f)\right) \leq \tau\left(S_{c, n-1}^{\frac{1}{2}}(f) S_{c, n}^{p-1}(f) S_{c, n-1}^{\frac{1}{2}}(f)\right) .
$$


The second term can be deduced from the nontrivial duality results in Lemma 2.3 for $1<p<\infty$ as follows:

$$
\begin{aligned}
B^{2} & =\sum_{k} \tau \int S_{c, k}^{2-p}(f)-S_{c, k-1}^{2-p}(f) \sum_{n \geq k} \sum_{|I|=2^{-n+1}} \frac{\left|\left\langle\varphi, w_{I}\right\rangle\right|^{2}}{|I|} \mathbb{1}_{I} \\
& =\sum_{k} \tau \sum_{j} S_{c, k}^{2-p}(f)-S_{c, k-1}^{2-p}(f) \int_{I_{k}^{j}} \sum_{n \geq k} \sum_{|I|=2^{-n+1}} \frac{\left|\left\langle\varphi, w_{I}\right\rangle\right|^{2}}{|I|} \mathbb{1}_{I} \\
& =\sum_{k} \tau \sum_{j} \int \mathbb{1}_{I_{k}^{j}}(x) S_{c, k}^{2-p}(f)(x)-S_{c, k-1}^{2-p}(f)(x) \frac{1}{\left|I_{k}^{j}\right|} \sum_{I \subset I_{k}^{j}}\left|\left\langle\varphi, w_{I}\right\rangle\right|^{2} d x \\
& =\sum_{k} \tau \int S_{c, k}^{2-p}(f)(x)-S_{c, k-1}^{2-p}(f)(x) \frac{1}{\left|I_{k}^{x}\right|} \sum_{I \subset I_{k}^{x}}\left|\left\langle\varphi, w_{I}\right\rangle\right|^{2} d x \\
& \leq\left\|\sum_{k} S_{c, k}^{2-p}(f)-S_{c, k-1}^{2-p}(f)\right\|_{L_{\left(p^{\prime} / 2\right)^{\prime}}}\left\|\sup _{k} \frac{1}{\left|I_{k}^{x}\right|} \sum_{I \subset I_{k}^{x}}\left|\left\langle\varphi, w_{I}\right\rangle\right|^{2}\right\|_{L_{p^{\prime} / 2}} \\
& =\|\varphi\|_{L_{p^{\prime}}^{c} \mathcal{M O O}}^{2}\|f\|_{\mathcal{H}_{p}^{c}}^{2-p} .
\end{aligned}
$$

The first equality uses the Fubini theorem, and the second one uses the fact that $S_{c, k-1}(f)$ and $S_{c, k}(f)$ are constant on the dyadic intervals with length $2^{-k+1}$.

For the other direction, we can carry out the proof as in the case $p=1$. Suppose that $l$ is a bounded linear functional on $\mathcal{H}_{p}^{c}(\mathbb{R}, \mathcal{M})$. By the embedding operator $\Phi$, by the Hahn-Banach theorem, and by the results in Lemma 2.1, we can find $g=\left(g_{I}\right)_{I \in \mathcal{D}}$ such that $\|g\|_{L_{p^{\prime}}\left(\mathcal{N} ; \ell_{2}^{c}(\mathcal{D})\right)}=\|l\|$ and

$$
l(f)=\tau \int \sum_{I \in \mathcal{D}} g_{I}^{*} \frac{\left\langle f, w_{I}\right\rangle}{|I|^{\frac{1}{2}}} \mathbb{1}_{I}, \forall f \in S_{\mathcal{N}} .
$$

Now let $\varphi=\Psi(g)$ be as defined in (2.11). The orthogonality of the $w_{I}$ 's yields

$$
\begin{aligned}
\| \sup _{n}^{+} & \frac{1}{\left|I_{n}^{x}\right|} \sum_{I \subset I_{n}^{x}}\left|\left\langle\varphi, w_{I}\right\rangle\right|^{2}\left\|_{L_{p^{\prime} / 2}(\mathcal{N})}=\right\| \sup _{n}+\frac{1}{\left|I_{n}^{x}\right|} \sum_{I \subset I_{n}^{x}}\left|\int \frac{g_{I}}{|I|^{\frac{1}{2}}} \mathbb{1}_{I}\right|^{2} \|_{L_{p^{\prime} / 2}(\mathcal{N})} \\
& \leq\left\|\sup _{n}+\frac{1}{\left|I_{n}^{x}\right|} \sum_{I \subset I_{n}^{x}} \int_{I_{n}^{x}}\left|g_{I}\right|^{2}\right\|_{L_{p^{\prime} / 2}(\mathcal{N})} \leq\left\|\sup _{n}+\frac{1}{\left|I_{n}^{x}\right|} \int_{I_{n}^{x}} \sum_{I \subset I_{n}^{x}}\left|g_{I}\right|^{2}\right\|_{L_{p^{\prime} / 2}(\mathcal{N})} \\
& \leq\left\|\sup _{n}^{+} \frac{1}{\left|I_{n}^{x}\right|} \int_{I_{n}^{x}} \sum_{I \in \mathcal{D}}\left|g_{I}\right|^{2}\right\|_{L_{p^{\prime} / 2}(\mathcal{N})} \leq c\left\|\sum_{I \in \mathcal{D}}\left|g_{I}\right|^{2}\right\|_{L_{p^{\prime} / 2}(\mathcal{N})} \\
& =c\left\|\left(g_{I}\right)_{I}\right\|_{L_{p^{\prime}}\left(\mathcal{N} ; \ell_{2}^{c}(\mathcal{D})\right)},
\end{aligned}
$$

where for the first inequality we have used the Kadison-Schwarz inequality, and the last inequality is (3.1). Also due to the orthogonality of the $w_{I}$ 's, we get

$$
l(f)=\tau \int \sum_{I \in \mathcal{D}} g_{I}^{*} \frac{\left\langle f, w_{I}\right\rangle}{|I|^{\frac{1}{2}}} \mathbb{1}_{I}=\tau \int \varphi^{*} f,
$$

for all $f \in S_{\mathcal{N}}$. Therefore, we have completed the proof. 
Instead of using the noncommutative Doob's inequality, we will use the following noncommutative Stein inequality from [20] to prove the duality between the spaces $\mathcal{H}_{p}^{c}, 1<p<\infty$.

Let $\left(\mathcal{E}_{n}\right)_{n}$ be the conditional expectation with respect to a filtration $\left(\mathcal{N}_{n}\right)_{n}$ of $\mathcal{N}$.

Lemma 3.5. Let $1<p<\infty$ and $a=\left(a_{n}\right)_{n} \in L_{p}\left(\mathcal{N} ; \ell_{2}^{c}\right)$. Then there exists a constant depending only on $p$ such that

$$
\left\|\left(\sum_{n}\left|\mathcal{E}_{n} a_{n}\right|^{2}\right)^{\frac{1}{2}}\right\|_{L_{p}(\mathcal{N})} \leq c_{p}\left\|\left(\sum_{n}\left|a_{n}\right|^{2}\right)^{\frac{1}{2}}\right\|_{L_{p}(\mathcal{N})} .
$$

Theorem 3.6. For any $1<p<\infty$, we have

$$
\left(\mathcal{H}_{p}^{c}(\mathbb{R}, \mathcal{M})\right)^{*}=\mathcal{H}_{p^{\prime}}^{c}(\mathbb{R}, \mathcal{M}) .
$$

Proof. By a reasoning similar to that in the proof of Theorem 3.2, we calculate

$$
\begin{aligned}
\left|l_{\varphi}(f)\right| & =\left|\tau \int \varphi^{*} f d x\right|=\left|\sum_{n} \tau \int \sum_{|I|=2^{-n+1}}\left\langle\varphi, w_{I}\right\rangle^{*} w_{I} \sum_{\left|I^{\prime}\right|=2^{-n+1}}\left\langle f, w_{I^{\prime}}\right\rangle w_{I^{\prime}} d x\right| \\
& =\left|\sum_{n} \tau \int \sum_{|I|=2^{-n+1}} \frac{\left\langle\varphi, w_{I}\right\rangle^{*}}{|I|^{\frac{1}{2}}} \mathbb{1}_{I} \frac{\left\langle f, w_{I}\right\rangle}{|I|^{\frac{1}{2}}} \mathbb{1}_{I} d x\right| \\
& \leq\left\|\left(\sum_{I \in \mathcal{D}} \frac{\left|\left\langle f, w_{I}\right\rangle\right|^{2}}{|I|} \mathbb{1}_{I}\right)^{\frac{1}{2}}\right\|_{L_{p}(\mathbb{R}, \mathcal{M})} \cdot\left\|\left(\sum_{I \in \mathcal{D}} \frac{\left|\left\langle\varphi, w_{I}\right\rangle\right|^{2}}{|I|} \mathbb{1}_{I}\right)^{\frac{1}{2}}\right\|_{L_{p^{\prime}}(\mathbb{R}, \mathcal{M})^{*}}
\end{aligned}
$$

Now, we turn to the proof of the inverse direction. Take a bounded linear functional $l \in\left(\mathcal{H}_{p}^{c}(\mathbb{R}, \mathcal{M})\right)^{*}$. By the embedding operator $\Phi$ and the Hahn-Banach extension theorem, $l$ extends to a bounded linear functional on $L_{p}\left(\mathcal{N} ; \ell_{2}^{c}\right)$ with the same norm. Thus by (2.1), there exists a sequence $g=\left(g_{I}\right)_{I}$ such that

$$
\|g\|_{L_{q}\left(\mathcal{N} ; l_{2}^{c}(\mathcal{D})\right)}=\|l\|
$$

and

$$
l(f)=\tau \int \sum_{I \in \mathcal{D}} g_{p}^{*} \frac{\left\langle f, w_{I}\right\rangle}{|I|^{\frac{1}{2}}} \mathbb{1}_{I}, \forall f \in S_{\mathcal{N}} .
$$

Now let $\varphi=\Psi(g)$ where $\Psi$ is defined in (2.11). Then, applying the Stein inequality (3.5) to the conditional expectation

$$
\mathcal{E}_{I}(h)=\sum_{J} \frac{1}{|J|} \int_{J} h(y) d y \cdot \mathbb{1}_{J},
$$

where $J$ is a dyadic interval with the same length as $I$, we get

$$
\begin{aligned}
\|\varphi\|_{\mathcal{H}_{p^{\prime}}^{c}(\mathbb{R}, \mathcal{M})} & =\left\|\left(\sum_{I \in \mathcal{D}}\left|\frac{1}{|I|} \int_{I} g_{I} d y \cdot \mathbb{1}_{I}\right|^{2}\right)^{\frac{1}{2}}\right\|_{L_{p^{\prime}}(\mathcal{N})} \\
& \leq\left\|\left(\sum_{I \in \mathcal{D}}\left|\mathcal{E}_{I}\left(g_{I}\right)\right|^{2}\right)^{\frac{1}{2}}\right\|_{L_{p^{\prime}}(\mathcal{N})} \leq c_{p^{\prime}}\left\|\left(\sum_{I \in \mathcal{D}}\left|g_{I}\right|^{2}\right)^{\frac{1}{2}}\right\|_{L_{p^{\prime}}(\mathcal{N})} .
\end{aligned}
$$


By the orthogonality of the $w_{I}$ 's, we have

$$
l(f)=\tau \int \sum_{I \in \mathcal{D}} g_{I}^{*} \frac{\left\langle f, w_{I}\right\rangle}{|I|^{\frac{1}{2}}} \mathbb{1}_{I}=\tau \int \varphi^{*} f
$$

for all $f \in S_{\mathcal{N}}$.

From the proof of the second part of Theorem 3.2, Theorem 3.3 and Theorem 3.6, we obtain the boundedness of $\Psi$, which we state as a corollary:

Corollary 3.7. (i) Let $1<p<\infty$. Then $\Psi$ is a projection map from $L_{p}\left(\mathcal{N} ; \ell_{2}^{c}(\mathcal{D})\right)$ onto $\mathcal{H}_{p}^{c}(\mathbb{R}, \mathcal{M})$ if we identify the latter as a subspace of the former.

(ii) For $2<p \leq \infty, \Psi$ is a bounded map from $L_{p}\left(\mathcal{N} ; \ell_{2}^{c}(\mathcal{D})\right)$ to $L_{p}^{c} \mathcal{M O}(\mathbb{R}, \mathcal{M})$.

Theorem 3.3 and Theorem 3.6 immediately imply the following corollary:

Corollary 3.8. Let $2<p<\infty$. Then

$$
\mathcal{H}_{p}^{c}(\mathbb{R}, \mathcal{M})=L_{p}^{c} \mathcal{M O}(\mathbb{R}, \mathcal{M}), \quad \forall 2<p<\infty,
$$

with equivalent norms.

\section{Interpolation}

This section is devoted to interpolation for our wavelet Hardy spaces. The interpolation results below will be needed in the next section to compare our Hardy spaces with those of Mei.

Lemma 4.1. Let $1<p_{0}<p<p_{1}<\infty$. We have

$$
\left[\mathcal{H}_{p_{0}}^{c}(\mathbb{R}, \mathcal{M}), \mathcal{H}_{p_{1}}^{c}(\mathbb{R}, \mathcal{M})\right]_{\theta}=\mathcal{H}_{p}^{c}(\mathbb{R}, \mathcal{M})
$$

with equivalent norms, where $\theta$ satisfies $\frac{1}{p}=\frac{1-\theta}{p_{0}}+\frac{\theta}{p_{1}}$.

Proof. The embedding map $\Phi$ yields

$$
\left[\mathcal{H}_{p_{0}}^{c}, \mathcal{H}_{p_{1}}^{c}\right]_{\theta} \subset \mathcal{H}_{p}^{c} .
$$

On the other hand, it is the projection map $\Psi$ from $L_{p}\left(\mathcal{N} ; \ell_{2}^{c}(\mathcal{D})\right)$ onto $\mathcal{H}_{p}^{c}(\mathbb{R}, \mathcal{M})$ stated in Corollary 3.7 that yields the inverse direction.

Theorem 4.2. Let $1 \leq q<p<\infty$. We have

$$
\left[\mathcal{B M O}^{c}(\mathbb{R}, \mathcal{M}), \mathcal{H}_{q}^{c}(\mathbb{R}, \mathcal{M})\right]_{\frac{q}{p}}=\mathcal{H}_{p}^{c}(\mathbb{R}, \mathcal{M})
$$

with equivalent norms. 
Proof. We will prove the theorem using the same general strategy as in [18].

Step 1. We prove the conclusion for $2<q<p<\infty$. The identity can be seen easily from the following two inclusions. On the one hand, the operator $\Phi$ from (2.10), together with (2.2), yields

$$
\left[\mathcal{H}_{1}^{c}(\mathbb{R}, \mathcal{M}), \mathcal{H}_{q^{\prime}}^{c}(\mathbb{R}, \mathcal{M})\right]_{\frac{q}{p}} \subset \mathcal{H}_{p^{\prime}}^{c}(\mathbb{R}, \mathcal{M}) .
$$

Then by duality and Corollary 3.8, we have

$$
L_{p}^{c} \mathcal{M O}(\mathbb{R}, \mathcal{M}) \subset\left[\mathcal{B} \mathcal{M O} \mathcal{O}^{c}(\mathbb{R}, \mathcal{M}), L_{q}^{c} \mathcal{M O}(\mathbb{R}, \mathcal{M})\right]_{\frac{q}{p}}
$$

On the other hand, the operator $\mathcal{T}$ identifying $L_{p}^{c} \mathcal{M O}(\mathbb{R}, \mathcal{M})$ as a subspace of $L_{p}\left(L_{\infty}\left(\mathcal{N} \bar{\otimes} B\left(\ell_{2}(\mathcal{D})\right) ; \ell_{\infty}^{c}\right)\right.$ and defined by

$$
\mathcal{T}(\varphi)=\left\langle f, w_{I}\right\rangle\left|I_{k}^{t}\right|^{-\frac{1}{2}} \mathbb{1}_{I \subset I_{k}^{t}}(I) \otimes e_{I, 1},
$$

together with Lemma 2.4, yields

$$
\left[\mathcal{B M O}^{c}(\mathbb{R}, \mathcal{M}), L_{q}^{c} \mathcal{M O}(\mathbb{R}, \mathcal{M})\right]_{\frac{q}{p}} \subset L_{p}^{c} \mathcal{M O}(\mathbb{R}, \mathcal{M})
$$

Step 2. We prove the conclusion for $1<q<p<\infty$. This step can be divided into two substeps.

Substep 2.1: $p>2$. Let $p<s<\infty$. By Step 1, we have

$$
\left[\mathcal{B M O}^{c}(\mathbb{R}, \mathcal{M}), \mathcal{H}_{p}^{c}(\mathbb{R}, \mathcal{M})\right]_{\frac{p}{s}}=\mathcal{H}_{s}^{c}(\mathbb{R}, \mathcal{M}) .
$$

On the other hand, by Theorem 4.1, we have

$$
\left[\mathcal{H}_{q}^{c}, \mathcal{H}_{s}^{c}\right]_{\theta}=\mathcal{H}_{p}^{c}
$$

where here (and in the rest of the paper) $\theta$ denotes the interpolation parameter. Then Wolff's interpolation theorem yields the result.

Substep 2.2: $p \leq 2$. Let $s>2$, then by Substep 2.1, we have

$$
\left[\mathcal{B M O}^{c}(\mathbb{R}, \mathcal{M}), \mathcal{H}_{p}^{c}(\mathbb{R}, \mathcal{M})\right]_{\frac{p}{s}}=\mathcal{H}_{s}^{c}(\mathbb{R}, \mathcal{M}) .
$$

Together with Lemma 4.1, Wolff's interpolation theorem yields the result.

Step 3. We prove the conclusion for $1=q<p<\infty$. Take $s>\max (p, 2)$. By Step 2 and duality (see Theorem 4.3.1 in [2]), we get

$$
\left[\mathcal{H}_{1}^{c}, \mathcal{H}_{s}^{c}\right]_{\theta}=\mathcal{H}_{p}^{c}
$$

Then together with Step 2, Wolff's interpolation yields the conclusion.

Theorem 4.3. For $1<p<\infty$, we have

$$
\mathcal{H}_{p}(\mathbb{R}, \mathcal{M})=L_{p}(\mathcal{N})
$$

with equivalent norms. 
Proof. There are several ways to prove this result. One can prove it by the strategy used in [20] together with Stein's inequality (3.5). Here, we just use that $L_{p}(\mathcal{M})$ with $1<p<\infty$ is a UMD space and our $\left(w_{I}\right)_{I}$ is a complete orthonormal basis.

So, by Theorem 3.8 in [7], we have

$$
\|f\|_{L_{p}(\mathcal{N})} \simeq\left(\mathbb{E}\left\|\sum_{I \in \mathcal{D}} \varepsilon_{I} \frac{\left\langle f, w_{I}\right\rangle}{|I|^{\frac{1}{2}}} \mathbb{1}_{I}\right\|_{L_{p}(\mathcal{N})}^{p}\right)^{\frac{1}{p}} .
$$

We complete the proof for $2 \leq p<\infty$ by Khintchine's inequalities. Now, let us prove the case $1<p<2$. Let $f \in \mathcal{H}_{p}(\mathbb{R}, \mathcal{M})$, then for any $\epsilon>0$, by the definition of $\mathcal{H}_{p}(\mathbb{R}, \mathcal{M})$, there exists a decomposition $f=f_{c}+f_{r}$ such that

$$
\left\|f_{c}\right\|_{\mathcal{H}_{p}^{c}(\mathbb{R}, \mathcal{M})}+\left\|f_{r}\right\|_{\mathcal{H}_{p}^{r}(\mathbb{R}, \mathcal{M})} \leq\|f\|_{\mathcal{H}_{p}(\mathbb{R}, \mathcal{M})}+\epsilon .
$$

Take any $g \in L_{p^{\prime}}(\mathcal{N})$, by the results for $p^{\prime}>2$, the operator-valued Calderón identity (2.5) yields

$$
\begin{aligned}
\left|\tau \int g f^{*}\right| & =\left|\sum_{I \in \mathcal{D}} \tau \int \frac{\left\langle g, w_{I}\right\rangle}{|I|^{\frac{1}{2}}} \mathbb{1}_{I} \cdot \frac{\left\langle f^{*}, w_{I}\right\rangle}{|I|^{\frac{1}{2}}} \mathbb{1}_{I}\right| \\
& \leq\left|\sum_{I \in \mathcal{D}} \tau \int \frac{\left\langle g, w_{I}\right\rangle}{|I|^{\frac{1}{2}}} \mathbb{1}_{I} \cdot \frac{\left\langle f_{c}^{*}, w_{I}\right\rangle}{|I|^{\frac{1}{2}}} \mathbb{1}_{I}\right|+\left|\sum_{I \in \mathcal{D}} \tau \int \frac{\left\langle g, w_{I}\right\rangle}{|I|^{\frac{1}{2}}} \mathbb{1}_{I} \cdot \frac{\left\langle f_{r}^{*}, w_{I}\right\rangle}{|I|^{\frac{1}{2}}} \mathbb{1}_{I}\right| \\
& \leq\left\|S_{c}(g)\right\|_{L_{p^{\prime}}(\mathcal{N})}\left\|S_{c}\left(f_{c}\right)\right\|_{L_{p}(\mathcal{N})}+\mid S_{r}(g)\left\|_{L_{p^{\prime}}(\mathcal{N})}\right\| S_{r}\left(f_{r}\right) \|_{L_{p}(\mathcal{N})} \\
& \leq c_{p^{\prime}}\|g\|_{L_{p^{\prime}}}\left(\|f\|_{\mathcal{H}_{p}(\mathbb{R}, \mathcal{M})}+\epsilon\right) .
\end{aligned}
$$

Taking the sup and letting $\epsilon \rightarrow 0$, we get the desired result.

Finally, we prove the inverse inequality. Let $f \in L_{p}(\mathcal{N})$. By duality, we can find two sequences of functions $\left(F_{c, I}\right)_{I} \in L_{p}\left(\mathcal{N} ; \ell_{2}^{c}(\mathcal{D})\right)$ and $\left(F_{r, I}\right)_{I} \in L_{p}\left(\mathcal{N} ; \ell_{2}^{r}(\mathcal{D})\right)$ such that $F_{c, I}+F_{r, I}=\left\langle f, w_{I}\right\rangle|I|^{-\frac{1}{2}} \mathbb{1}_{I}$ and

$$
\left\|\left(F_{c, I}\right)_{I}\right\|_{L_{p}\left(\mathcal{N} ; \ell_{2}^{c}(\mathcal{D})\right)}+\left\|\left(F_{r, I}\right)_{I}\right\|_{L_{p}\left(\mathcal{N} ; \ell_{2}^{r}(\mathcal{D})\right)} \leq\|f\|_{L_{p}(\mathcal{N})} .
$$

Let $f_{c}=\Psi\left(\left(F_{c, I}\right)_{I}\right)$ and $f_{r}=\Psi\left(\left(F_{r, I}\right)_{I}\right)$. By identity $(2.5)$, we have $f=f_{c}+f_{r}$. On the other hand, by the Stein inequality (3.5), we have that $\left\|f_{c}\right\|_{\mathcal{H}_{p}^{c}(\mathbb{R}, \mathcal{M})} \leq$ $\left\|\left(F_{c, I}\right)_{I}\right\|_{L_{p}\left(\mathcal{N} ; \ell_{2}^{c}(\mathcal{D})\right)}$ and $\left\|f_{r}\right\|_{\mathcal{H}_{p}^{r}(\mathbb{R}, \mathcal{M})} \leq\left\|\left(F_{r, I}\right)_{I}\right\|_{L_{p}\left(\mathcal{N} ; \ell_{2}^{r}(\mathcal{D})\right)}$. So we have found the desired decomposition of $f$.

Theorem 4.4. The following results hold with equivalent norms:

(i) Let $1 \leq q<p<\infty$. We have

$$
\left[\mathcal{B M O}(\mathbb{R}, \mathcal{M}), L_{q}(\mathcal{N})\right]_{\frac{q}{p}}=L_{p}(\mathcal{N}) .
$$

(ii) Let $1<q<p \leq \infty$. We have

$$
\left[\mathcal{H}_{1}(\mathbb{R}, \mathcal{M}), L_{p}(\mathcal{N})\right]_{\frac{p^{\prime}}{q^{\prime}}}=L_{q}(\mathcal{N})
$$

(iii) Let $1<p<\infty$. We have

$$
\left[\mathcal{B} \mathcal{M O}(\mathbb{R}, \mathcal{M}), \mathcal{H}_{1}(\mathbb{R}, \mathcal{M})\right]_{\frac{1}{p}}=L_{p}(\mathcal{N}) .
$$


In order to prove this theorem, we need the following result from the theory of interpolation. We formulate it here without proof.

Lemma 4.5. Let $A_{0}, B_{0}, A_{1}$, and $B_{1}$ be four Banach spaces satisfying the property needed for interpolation. Then

$$
\left[A_{0}+B_{0}, A_{1}+B_{1}\right]_{\theta} \supset\left[A_{0}, A_{1}\right]_{\theta}+\left[B_{0}, B_{1}\right]_{\theta}
$$

and

$$
\left[A_{0} \cap B_{0}, A_{1} \cap B_{1}\right]_{\theta} \subset\left[A_{0}, A_{1}\right]_{\theta} \cap\left[B_{0}, B_{1}\right]_{\theta} .
$$

Proof of Theorem 4.4. (i) We use a strategy, similar to, but different from, that used to prove Theorem 4.2.

Step 1. We prove the results for $2 \leq q<p<\infty$. By Theorem 4.3, Theorem 4.2 and Lemma 4.5, we have

$$
\left[\mathcal{B M O}(\mathbb{R}, \mathcal{M}), L_{q}(\mathcal{N})\right]_{\frac{q}{p}} \subset L_{p}(\mathcal{N}) .
$$

The inverse direction follows from $L_{\infty}(\mathcal{N}) \subset \mathcal{B M O}(\mathbb{R}, \mathcal{M})$ :

$$
L_{p}(\mathcal{N})=\left[L_{\infty}(\mathcal{N}), L_{q}(\mathcal{N})\right]_{\frac{q}{p}} \subset\left[\mathcal{B M O}(\mathbb{R}, \mathcal{M}), L_{q}(\mathcal{N})\right]_{\frac{q}{p}} .
$$

Step 2. We prove the results for $1 \leq q<2 \leq p<\infty$. By Step 1, we have

$$
\left[\mathcal{B M O}(\mathbb{R}, \mathcal{M}), L_{2}(\mathcal{N})\right]_{\frac{2}{p}}=L_{p}(\mathcal{N}) .
$$

Together with

$$
L_{2}(\mathcal{N})=\left[L_{p}(\mathcal{N}), L_{q}(\mathcal{N})\right]_{\theta},
$$

Wolff's interpolation yields the conclusion.

Step 3. We prove the results for $1 \leq q<p<2$. By Step 2, we have

$$
\left[\mathcal{B M O}(\mathbb{R}, \mathcal{M}), L_{p}(\mathcal{N})\right]_{\frac{p}{2}}=L_{2}(\mathcal{N}) .
$$

Together with

$$
L_{p}(\mathcal{N})=\left[L_{2}(\mathcal{N}), L_{q}(\mathcal{N})\right]_{\theta},
$$

Wolff's interpolation yields the conclusion.

(ii) The results for $1<q<p<\infty$ can be immediately proved by duality and the partial results in $(i)$. For $p=\infty$, take $q<s<\infty$. Then, by Wolff's argument, we get the conclusion.

(iii) First, we prove the conclusion for $p<2$. By (i) and (ii), we have

$$
\left[\mathcal{B M O}(\mathbb{R}, \mathcal{M}), L_{p}(\mathcal{N})\right]_{\frac{p}{p^{\prime}}}=L_{p^{\prime}}(\mathcal{N}) \quad \text { and } \quad\left[\mathcal{H}_{1}(\mathbb{R}, \mathcal{M}), L_{p^{\prime}}(\mathcal{N})\right]_{\frac{p}{p^{\prime}}}=L_{p}(\mathcal{N})
$$

We end with Wolff's argument. The proof for $p>2$ is the same. Finally, when $p=2$, we can take $s>2$. By the results for $p \neq 2$ and the reiteration theorem (see Theorem 4.6 .1 in [2]), we get

$$
\begin{aligned}
L_{2} & \left.\left.=\left[L_{s}, L_{s^{\prime}}\right]_{\theta}=\left[\mathcal{B M O}(\mathbb{R}, \mathcal{M}), \mathcal{H}_{1}(\mathbb{R}, \mathcal{M})\right]_{\frac{1}{s}}, \mathcal{B} \mathcal{M O}(\mathbb{R}, \mathcal{M}), \mathcal{H}_{1}(\mathbb{R}, \mathcal{M})\right]_{\frac{1}{s^{\prime}}}\right]_{\theta} \\
& =\left[\mathcal{B M O}(\mathbb{R}, \mathcal{M}), \mathcal{H}_{1}(\mathbb{R}, \mathcal{M})\right]_{\theta} .
\end{aligned}
$$




\section{Comparison with Mei's results}

We denote by $H_{p}^{c}(\mathbb{R}, \mathcal{M})$ the column Hardy space defined in [15] through operatorvalued Lusin square function, and by $\operatorname{BMO}^{c}(\mathbb{R}, \mathcal{M})$ the column bounded mean oscillation space appearing in matrix-valued harmonic analysis (see, e.g., [15]). We have the following result:

Theorem 5.1. We have

$$
\mathcal{B M O}^{c}(\mathbb{R}, \mathcal{M})=\mathrm{BMO}^{c}(\mathbb{R}, \mathcal{M})
$$

with equivalent norms. Similar results holds for the row spaces. Consequently, $\mathcal{B M O}(\mathbb{R}, \mathcal{M})=\operatorname{BMO}(\mathbb{R}, \mathcal{M})$ with equivalent norms.

The theorem can be easily seen from the corresponding $\operatorname{BMO}(\mathbb{R}, H)$-spaces. However, we also use the idea in the proof of Theorem 1.2 in [7] to prove the theorem.

Proof. $\mathcal{B M O}^{c}(\mathbb{R}, \mathcal{M}) \subset \operatorname{BMO}^{c}(\mathbb{R}, \mathcal{M})$. Let $\varphi \in \mathcal{B M O}_{c}(\mathbb{R}, \mathcal{M})$. As in the beginning of the proof of Theorem 1.2 in [7], fix a finite interval $I \subset \mathbb{R}$, and consider the collections of dyadic intervals:

(1) $\mathcal{D}_{1}:=\{J \in \mathcal{D} ; 2|J|>|I|\}$,

(2) $\mathcal{D}_{2}:=\{J \in \mathcal{D} ; 2|J| \leq|I|, 2 J \cap 2 I=\emptyset\}$,

(3) $\mathcal{D}_{3}:=\{J \in \mathcal{D} ; 2|J| \leq|I|, 2 J \cap 2 I \neq \emptyset\}$.

Let $a_{J}=\left\langle\varphi, \omega_{J}\right\rangle$. Then we have an a priori formal series

$$
\varphi_{1}(x)=\sum_{J \in \mathcal{D}_{1}} a_{J}\left[\omega_{J}(x)-\omega_{J}\left(c_{I}\right)\right], \quad \varphi_{i}(x)=\sum_{J \in \mathcal{D}_{i}} a_{J} \omega_{J}(x), \quad i=2,3,
$$

where $c_{I}$ is the center of the interval $I$. Write $\varphi_{I}=\varphi_{1}+\varphi_{2}+\varphi_{3}$. By an argument similar to that in [7], we only need to prove

$$
\left\|\frac{1}{|I|} \int_{I}\left|\varphi_{I}(x)\right|^{2} d x\right\|_{\mathcal{M}}<\infty
$$

By scaling we can assume:

$$
\sup _{I} \frac{1}{|I|}\left\|\sum_{J \subset I}\left|a_{J}\right|^{2}\right\|=1
$$

Then we have the obvious bound $\left\|a_{J}\right\| \leq|J|^{\frac{1}{2}}$ for the individual terms.

We have the following estimates for $\varphi_{1}$ :

$$
\begin{aligned}
\left\|\frac{1}{|I|} \int_{I}\left|\varphi_{1}(x)\right|^{2} d x\right\| & \leq \frac{1}{|I|}\left(\sum_{J \in \mathcal{D}_{1}}\left\|a_{J}\right\|\left|\omega_{J}(x)-\omega_{J}\left(c_{I}\right)\right|\right)^{2} d x \\
& \leq c \frac{1}{|I|} \int_{I}\left[\sum_{J \in \mathcal{D}_{1}}|J|^{\frac{1}{2}}|I||J|^{-\frac{3}{2}}\left(1+\frac{\operatorname{dist}(I, J)}{|J|}\right)^{-2}\right]^{2} d x \\
& =c\left[\sum_{j=0}^{\infty} \sum_{|J| \in\left(2^{j-1}, 2^{j}\right]|I|}|I||J|^{-1}\left(1+\frac{\operatorname{dist}(I, J)}{|J|}\right)^{-2}\right]^{2}<\infty .
\end{aligned}
$$


For $\varphi_{2}$,

$$
\begin{aligned}
\left\|\frac{1}{|I|} \int_{I}\left|\varphi_{2}(x)\right|^{2} d x\right\| & \leq \frac{1}{|I|} \int_{I}\left\|\sum_{\mathcal{D}_{2}} a_{J} \omega_{J}(x)\right\|^{2} d x \leq \frac{1}{|I|} \int_{I}\left(\sum_{\mathcal{D}_{2}}\left\|a_{J}\right\|\left|\omega_{J}(x)\right|\right)^{2} d x \\
& \leq c \frac{1}{|I|} \int_{I}\left[\sum_{\mathcal{D}_{2}}|J|^{\frac{1}{2}}|J|^{-\frac{1}{2}}\left(\frac{\operatorname{dist}(I, J)}{|J|}\right)^{-2}\right]^{2} d x \\
& =c\left[\sum_{j=1}^{\infty} \sum_{|J| \in\left(2^{-j-1}, 2^{-j}\right)|I|, \operatorname{dist}(I, J)>2^{-1}|I|}\left(\frac{\operatorname{dist}(I, J)}{|J|}\right)^{-2}\right]^{2}<\infty .
\end{aligned}
$$

And for $\varphi_{3}$,

$$
\left\|\frac{1}{|I|} \int_{I}\left|\varphi_{3}(x)\right|^{2} d x\right\| \leq \frac{1}{|I|}\left\|\sum_{J \in \mathcal{D}_{3}}\left|a_{J}\right|^{2}\right\| \leq \frac{1}{|I|}\left\|\sum_{J \subset 4 I}\left|a_{J}\right|^{2}\right\|<\infty .
$$

Hence, we deduce that:

$$
\left\|\int_{I}\left|\varphi_{I}(x)\right|^{2} d x\right\|_{\mathcal{M}} \leq c \sum_{i=1}^{3}\left\|\int_{I}\left|\varphi_{i}(x)\right|^{2} d x\right\|_{\mathcal{M}} \leq c|I| .
$$

Now we turn to the proof of the inverse direction, $\operatorname{BMO}^{c}(\mathbb{R}, \mathcal{M}) \subset \mathcal{B M} \mathcal{O}^{c}(\mathbb{R}, \mathcal{M})$. Let $\varphi \in \operatorname{BMO}^{c}(\mathbb{R}, \mathcal{M})$. The proof is very similar to that of Lemma 4.1 in Mei's work [15]. For any dyadic interval $I \subset \mathbb{R}$, write $\varphi=\varphi_{1}+\varphi_{2}+\varphi_{3}$, where $\varphi_{1}=\left(\varphi-\varphi_{2 I}\right) \chi_{2 I}, \varphi_{2}=\left(\varphi-\varphi_{2 I}\right) \chi_{2 I^{c}}, \varphi_{3}=\varphi_{2 I}$.

Thus

$$
\sum_{J \subset I}\left|\left\langle\varphi, \omega_{J}\right\rangle\right|^{2} \leq 2\left(\sum_{J \subset I}\left|\left\langle\varphi_{1}, \omega_{J}\right\rangle\right|^{2}+\sum_{J \subset I}\left|\left\langle\varphi_{2}, \omega_{J}\right\rangle\right|^{2}\right) .
$$

We have the following estimates for $\varphi_{1}$ :

$$
\left\|\sum_{J \subset I}\left|\left\langle\varphi_{1}, \omega_{J}\right\rangle\right|^{2}\right\| \leq\left\|\int\left|\varphi_{1}(x)\right|^{2} d x\right\| \leq c\left\|\int_{2 I}\left|\varphi-\varphi_{2 I}\right|^{2}\right\| \leq c|I| .
$$

And for $\varphi_{2}$,

$$
\begin{aligned}
\left\|\sum_{J \subset I}\left|\left\langle\varphi_{2}, \omega_{J}\right\rangle\right|^{2}\right\| & =\left\|\sum_{J \subset I}\left|\sum_{k=1}^{\infty} \int_{2^{k+1} I / 2^{k} I} \varphi_{2} \omega_{J} d x\right|^{2}\right\| \\
& \leq\left\|\sum_{J \subset I}\left(\sum_{k=1}^{\infty} \frac{1}{2^{2 k}} \int_{2^{k+1} I / 2^{k} I}\left|\varphi_{2}\right|^{2}\right)\left(\sum_{k=1}^{\infty} 2^{2 k} \int_{2^{k+1} I / 2^{k} I}\left|\omega_{J}\right|^{2}\right)\right\| \\
& \leq c\left(\sum_{k=1}^{\infty} \frac{1}{2^{2 k}}\left\|\int_{2^{k+1} I}\left|\varphi-\varphi_{2 I}\right|^{2}\right\|\right)\left(\sum_{J \subset I} \sum_{k=1}^{\infty} 2^{2 k} \int_{2^{k+1} I / 2^{k} I}\left|\omega_{J}\right|^{2}\right) \\
& \leq c|I|\|\varphi\|_{\mathcal{B} \mathcal{M O}_{c}}^{2} \sum_{j=0}^{\infty} 2^{j} \sum_{k=1}^{\infty} \int_{2^{k+1} I / 2^{k} I} \frac{2^{2 k} \frac{\left|2^{-j} I\right|^{3}}{\left|2^{k} I\right|^{4}} \leq c|I| .}{}
\end{aligned}
$$

Therefore, $\left\|\sum_{J \subset I}\left|\left\langle\varphi, \omega_{J}\right\rangle\right|^{2}\right\| \leq c|I|$, which completes our proof. 
Combined with Theorem 3.3 and Theorem 4.2, we have the following corollary:

Corollary 5.2. For $1 \leq p<\infty$, we have

$$
\mathcal{H}_{p}^{c}(\mathbb{R}, \mathcal{M})=H_{p}^{c}(\mathbb{R}, \mathcal{M}) .
$$

Similar results hold for $\mathcal{H}_{p}^{r}$ and $H_{p}^{r}$, and for $\mathcal{H}_{p}$ and $H_{p}$.

If $\mathcal{M}=\mathbb{C}, \mathcal{H}_{1}(\mathbb{R}, \mathbb{C})$ is just the usual Hardy space $H_{1}(\mathbb{R})$ on $\mathbb{R}$. $H_{1}(\mathbb{R})$ also has the following characterization:

$$
H_{1}(\mathbb{R})=\left\{f \in L_{1}(\mathbb{R}): H(f) \in L_{1}(\mathbb{R})\right\},
$$

where $H$ is the Hilbert transform. For any $f \in H_{1}(\mathbb{R})$,

$$
\|f\|_{H_{1}(\mathbb{R})} \approx\|f\|_{L_{1}(\mathbb{R})}+\|H(f)\|_{L_{1}(\mathbb{R})} .
$$

Thus $H_{1}(\mathbb{R})$ can be viewed as a subspace of $L_{1}(\mathbb{R}) \oplus_{1} L_{1}(\mathbb{R})$. The latter direct sum has its natural operator structure as an $L_{1}$ space. This induces an operator space structure on $H_{1}(\mathbb{R})$. Although $\left(w_{I}\right)_{I \in \mathcal{D}}$ is an unconditional basis of $H_{1}(\mathbb{R})$, Ricard $[22]$ (see also [23]) proved that $H_{1}(\mathbb{R})$ does not have a complete unconditional basis. However, in noncommutative analysis, one can introduce another natural operator space structure on $H_{1}(\mathbb{R})$ as follows: $S_{1}\left(H_{1}(\mathbb{R})\right)=\mathcal{H}_{1}\left(\mathbb{R}, B\left(\ell_{2}\right)\right)$, where $S_{1}$ is the trace class on $\ell_{2}$. Then we have the following result. Note that Ricard [23] obtained a similar result using Hilbert space techniques.

Corollary 5.3. The complete orthogonal system $\left(w_{I}\right)_{I \in \mathcal{D}}$ of $L_{2}(\mathbb{R})$ is a completely unconditional basis for $H_{1}(\mathbb{R})$ if we define the operator space structure imposed on $H_{1}(\mathbb{R})$ by $\mathcal{S}_{1}\left(H_{1}(\mathbb{R})\right)=\mathcal{H}_{1}\left(\mathbb{R}, B\left(\ell_{2}\right)\right)$.

Proof. Fix a finite subset $\mathcal{I} \subset \mathcal{D}$. Let $T_{\varepsilon} f:=\sum_{I \in \mathcal{I}} \varepsilon_{I}\left\langle f, w_{I}\right\rangle w_{I}$, where $\varepsilon_{I}= \pm 1$. By the definition of $\mathcal{H}_{1}^{c}(\mathbb{R}, \mathcal{M})$, the orthogonality of $\left(w_{I}\right)_{I \in \mathcal{D}}$ yields immediately that

$$
\begin{aligned}
\left\|T_{\varepsilon} f\right\|_{\mathcal{H}_{1}^{c}} & =\left\|\left(\sum_{I \in \mathcal{I}} \frac{\left|\left\langle f, w_{I}\right\rangle\right|^{2}}{|I|} \mathbb{1}_{I}(x)\right)^{\frac{1}{2}}\right\|_{L_{1}(\mathcal{N})} \\
& \leq\left\|\left(\sum_{I \in \mathcal{D}} \frac{\left|\left\langle f, w_{I}\right\rangle\right|^{2}}{|I|} \mathbb{1}_{I}(x)\right)^{\frac{1}{2}}\right\|_{L_{1}(\mathcal{N})}=\|f\|_{\mathcal{H}_{1}^{c}} .
\end{aligned}
$$

Similarly, the above inequality holds for $\mathcal{H}_{1}^{r}(\mathbb{R}, \mathcal{M})$. Now, let $f \in \mathcal{H}_{1}(\mathbb{R}, \mathcal{M})$. Then, for any $\epsilon>0$, there exists a decomposition $f=g+h$ such that

$$
\|g\|_{\mathcal{H}_{1}^{c}(\mathbb{R}, \mathcal{M})}+\|h\|_{\mathcal{H}_{1}^{r}(\mathbb{R}, \mathcal{M})} \leq\|f\|_{\mathcal{H}_{1}(\mathbb{R}, \mathcal{M})}+\epsilon .
$$

Therefore

$$
\begin{aligned}
\left\|T_{\varepsilon} f\right\|_{\mathcal{H}_{1}(\mathbb{R}, \mathcal{M})} & \leq\left\|T_{\varepsilon} g\right\|_{\mathcal{H}_{1}^{c}(\mathbb{R}, \mathcal{M})}+\left\|T_{\varepsilon} h\right\|_{\mathcal{H}_{1}^{c}(\mathbb{R}, \mathcal{M})} \\
& \leq\|g\|_{\mathcal{H}_{1}^{c}(\mathbb{R}, \mathcal{M})}+\|h\|_{\mathcal{H}_{1}^{r}(\mathbb{R}, \mathcal{M})} \leq\|f\|_{\mathcal{H}_{1}(\mathbb{R}, \mathcal{M})}+\epsilon .
\end{aligned}
$$

Letting $\epsilon \rightarrow 0$, we get the result. 


\section{References}

[1] Bekjan, T., Chen, Z., Perrin, M. And Yin, Z.: Atomic decomposition and interpolation for Hardy spaces of noncommutative martingales. J. Funct. Anal. 258 (2010), no. 7, 2483-2505.

[2] Bergh, J. And Löfström, J.: Interpolation spaces. An introduction. Grundlehren der Mathematischen Wissenschaften 223, Springer-Verlag, Berlin-New York, 1976.

[3] Cuculescu, I.: Martingales on von Neumann algebras. J. Multivariate Anal. 1 (1971), 17-27.

[4] Defant, A. And Junge, M.: Maximal theorems of Menchoff-Rademacher type in non-commutative $L_{q}$-spaces. J. Funct. Anal. 206 (2004), 322-355.

[5] Ferguson, S. H. And Lacey, M. T.: A characterization of product BMO by commutators. Acta Math. 189 (2002), no. 2, 143-160.

[6] Fefferman, C. And Stein, E. M.: $H^{p}$ spaces of several variables. Acta Math. 129 (1972), 137-193.

[7] Hytönen, T., Salinas, O. And Viviani, B.: Wavelet expansions for weighted vector-valued BMO functions. J. Anal. Math. 111 (2010), 321-337.

[8] Junge, M.: Doob's inequality for non-commutative martingales. J. Reine Angew. Math. 549 (2002), 149-190.

[9] Junge, M., Le Merdy, C. And Xu, Q.: $H^{\infty}$ functional calculus and square functions on noncommutative $L^{p}$-spaces. Astérisque 305 (2006).

[10] Junge, M. And Musat, M.: A noncommutative version of John-Nirenberg theorem. Trans. Amer. Math. Soc. 359 (2007), no. 1, 115-142.

[11] Junge, M. And Xu, Q.: Noncommutative Burkholder/Rosenthal inequalities. Ann. Probab. 31 (2003), no. 2, 948-995.

[12] Junge, M. And Xu, Q.: On the best constants in some non-commutative martingale inequalities. Bull. London Math. Soc. 37 (2005), 243-253.

[13] Junge, M. And Xu, Q.: Noncommutative maximal ergodic theorems. J. Amer. Math. Soc. 20 (2006), 385-439.

[14] Junge, M. And Xu, Q.: Noncommutative Burkholder/Rosenthal inequalities. II. Applications. Israel J. Math. 167 (2008), 227-282.

[15] MeI, T.: Operator valued Hardy spaces. Mem. Amer. Math. Soc. 188 (2007).

[16] Meyer, Y.: Wavelets and operators. Cambridge Studies in Advanced Mathematics 37, Cambridge University Press, Cambridge, 1992.

[17] Muscalu, C., Pipher, J., Tao, T. and Thiele, C.: Bi-parameter paraproducts. Acta Math. 193 (2004), 269-296.

[18] Musat, M.: Interpolation between non-commutative BMO and non-commutative $L_{p}$-spaces. J. Funct. Anal. 202 (2003), no. 1, 195-225.

[19] Pisier, G.: Non-commutative vector valued $L_{p}$-spaces and completely $p$-summing maps. Astérisque 247 (1998).

[20] Pisier, G. And Xu, Q: Non-commutative martingale inequalities. Comm. Math. Phys. 189 (1997), 667-698.

[21] Pisier, G. And Xu, Q.: Non-commutative $L_{p}$-spaces. In Handbook of the geometry of Banach spaces, Vol. 2, 1459-1517. North-Holland, Amsterdam, 2003. 
[22] Ricard, E.: L'espace $H^{1}$ n'a pas de base complètement inconditionnelle. C. R. Acad. Sci. Paris Sér. I Math. 331 (2000), 625-628.

[23] Ricard, E.: Décomposition de $H^{1}$, multiplicateurs de Schur et espaces d'operateurs. Thèse de Doctorat de l'Université Paris VI, 2001.

[24] WolfF, T.: A note on interpolation spaces. In Harmonic analysis (Minneapolis, Minn., 1981), 199-204. Lecture Notes in Mathematics 908, Springer, Berlin-New York, 1982.

Received April 26, 2011.

Guixiang Hong: Laboratoire de Mathématiques, Université de Franche-Comté, 25030 Besançon Cedex, France.

E-mail: guixiang.hong@univ-fcomte.fr

Zhi Yin: Laboratoire de Mathématiques, Université de Franche-Comté, 25030 Besançon Cedex, France.

School of Mathematics and Statistics, Wuhan University, Luo-Jia-Shan, Wuhan 430072, Hubei, China.

E-mail: hustyinzhi@163.com 